\title{
Comparaison des variants $\mathbf{A}$ et $\mathbf{C}$ de la caséine $\beta$ des laits de vaches Tarentaises en modèle fromager de type beaufort. II. Protéolyse et qualité des fromages
}

\author{
C Marie *, A Delacroix-Buchet \\ Station de recherches laitières, INRA, 78352 Jouy-en-Josas Cedex, France
}

(Reçu le 24 mai 1994; accepté le 22 août 1994)

\begin{abstract}
Résumé - Quinze minifabrications fromagères ont été réalisées avec des laits individuels de vaches Tarentaises caséine $\beta C C(\beta-C n C)$ ou $A A(\beta-C n A)$, selon une technologie pâte cuite pressée aussi voisine que possible de celle du fromage de Beaufort. Les minifromages (environ $220 \mathrm{~g}$ ) ont été affinés 8 semaines à $12^{\circ} \mathrm{C}$. La protéolyse, la structure, la texture et la flaveur des fromages $\beta$-Cn C ont été comparées à celles des fromages $\beta$-Cn A. II apparaît que les fromages $\beta-C n C$, qui ont une faible teneur en matière grasse et une structure irrégulière (observée au microscope électronique), se caractérisent par une texture plus ferme et moins élastique que les fromages $\beta$-Cn A. On retrouve, dans les minifromages, des différences dans l'hydrolyse de la caséine $\beta$ par la plasmine, liées à la structure biochimique particulière du variant $C$ : la cinétique de dégradation est plus lente (proportion de fraction $\gamma_{1}$ ) et les produits d'hydrolyse sont spécifiques. Les fromages $\beta-C n C$ ont un goût particulier et sont notés piquants plus souvent que les fromages $\beta$-Cn $A(P=0,13)$, par le jury d'analyses sensorielles. Le variant $C$ de la $\beta-C n$ confère donc une spécificité au fromage de Beaufort justifiant les termes du décret d'Appellation d'origine qui précise les races de vaches (Tarentaise et Abondance) du troupeau laitier destiné à la fabrication du fromage de Beaufort.
\end{abstract}

fromage / beaufort / caséine $\beta$ / variant génétique / Tarentaise / protéolyse / structure / texture et flaveur

Summary - $\beta$-casein $C$ and Beaufort-type cheese texture and quality. Fifteen cooked curd type cheeses were made at a laboratory scale with individual milk samples of Tarentaise cows (a local mountain breed) either with $\beta$-casein $C C(\beta-C n C)$ or with $A A(\beta-C n A)$ genotypes, using a technology as close as possible to the Beaufort-type cheese making. The small cheeses (about $220 \mathrm{~g}$ ) were ripened 8 weeks at $12^{\circ} \mathrm{C}$. Proteolysis, structure, texture, and flavour of the two types of cheese ( $\beta$-Cn $C \operatorname{or} \beta-C n A$ ) are compared. $\beta$-Cn C cheeses have a lower fat content and an irregular structure (as seen

* Stagiaire de DEA, ENSA, Rennes, France. Adresse actuelle : Station d'amélioration génétique des animaux, INRA Auzeville, BP 27, 31326 Castanet-Tolosan Cedex, France 
by electron microscopy) and thus, have a firmer and less elastic texture than $\beta$-Cn $A$ cheeses. We found, in the small cheeses, differences in the hydrolysis of $\beta$-Cn by plasmin in relation with the biochemical structure of the $C$ variant: the breakdown kinetics are slower (proportion of $\gamma_{1}$ fraction) and hydrolysis products are different. It ensues that $\beta-C n C$ cheeses have a specific flavour and in particular, are more often noted spicy $(\mathbb{P}=0.13)$ than the $\beta-C n$ A cheeses, by the panel of the sensory analyses. Thus, the $C$ variant of $\beta-C n$, and particularly, the Tarentaise breed of cows mentioned in the decree of Appellation d'Origine, gives a specificity to Beaufort cheese.

\section{cheese / Beaufort / $\beta$-casein / genetic variant / Tarentaise / proteolysis / structure / texture and flavour}

\section{INTRODUCTION}

Le beaufort est un fromage à pâte cuite pressée et à croûte morgée. Ce fromage traditionnel des Alpes ne peut être produit, d'après son décret d'Appellation d'origine, qu'avec du lait frais, cru et entier de vaches de races de Tarentaise ou d'Abondance. Les vaches Tarentaises représentent environ les 2 tiers du troupeau «Beaufort". Elles se caractérisent par une fréquence élevée $(17 \%)$ d'un variant rare de la caséine $\beta$ (le variant C) (Nuyts-Petit, 1991). Lors de la fabrication du fromage de Beaufort, la cuisson du caillé en cuve à une température comprise entre 54 et $56^{\circ} \mathrm{C}$ favorise l'égouttage du caillé et sélectionne les agents d'affinage. En effet, la flore coliforme du lait et l'enzyme coagulante apportée par la présure artisanale dite "sur recuite" sont détruites. Seuls subsistent les levains lactiques thermophiles principalement constitués de Lactobacillus helveticus et Lactobacillus delbrueckii subsp lactis apportés par la présure artisanale (Chamba et al, 1994) et le système plasminogène-plasmine du lait, d'origine sanguine, thermostable et activé par le chauffage en cuve (Grufferty et Fox, 1988). La protéolyse des fromages de Beaufort est donc essentiellement le fait de l'activité des lactobacilles thermophiles et de la plasmine. Cette dernière, appelée aussi protéase alcaline du lait, attaque préférentiellement les caséines $\alpha_{s 2}$ et $\beta$ (Le Bars et Gripon, 1989) au niveau des sites Lys-X ou Arg-X. Le variant $\beta$-Cn $C$ présente par rapport aux variants $A\left(A^{1}\right.$ ou $\left.A^{2}\right)$ fréquents en race Tarentaise, une substitution du résidu Glu 37 par un résidu Lys ce qui provoque la non phosphorylation du résidu Ser 35 (Grosclaude, 1988).

La $\beta$-Cn représente en moyenne $36 \%$ des caséines. Elle est hydrolysée en fractions $\gamma$ $\left(\gamma_{1}, \gamma_{2}, \gamma_{3}\right)$ et protéoses-peptones par la plasmine (Gordon et al, 1972 ; Kaminogawa et Yamauchi, 1972 ; Grufferty et Fox, 1988). Groves et al (1972) ont montré qu'aux variants $A$ et $B$ de la $\beta-C n$ correspondaient des variants $A$ et $B$ des fractions $\gamma$, mais ils n'ont pas signalé de fractions $\gamma \mathrm{C}$ correspondant à la $\beta-C n$ C. Papoff et al (1994) ont étudié et comparé l'hydrolyse par la plasmine de fractions purifiées de la $\beta-\mathrm{Cn} C$ et de la $\beta-C n A^{1}$. La séparation électrophorétique (gel PAGE à pH 8,6) des fractions d'hydrolysat insolubles à $\mathrm{pH} 4,6$ met en évidence les fractions $\gamma$ du variant $C$ : seule la fraction $\gamma_{1} C$ diffère de la fraction $\gamma_{1} A^{1}$ par sa faible mobilité liée à sa plus faible charge (non phosphorylation de la Ser 35). Le nombre de sites potentiels de coupure de la $\beta-C n C$ par la plasmine est supérieur à celui de la $\beta-C n A^{1}$ (11 contre 9) mais I'hydrolyse de la $\beta$-Cn $C$ est plus lente.

Un programme d'étude de l'incidence, en transformation fromagère, du variant $C$ de la $\beta-C n$ a été entrepris en 1989. Une première étape a consisté à comparer les caractéristiques physico-chimiques et rhéologiques (comportement à l'emprésurage) des laits possédant ou non le variant rare (le reste du génotype étant identique). Les 
résultats montrent l'originalité du variant $\mathrm{C}$ de la $\beta$-Cn (Nuyts-Petit, 1991). Dans une deuxième étape, on a réalisé à partir de laits individuels de vaches Tarentaises, collectés à la traite du matin dans les Alpes, des minifabrications de fromage de Beaufort, pour comparer des laits et des fromages homozygotes $\beta$-Cn $C$ à des laits et des fromages témoins homozygotes $\beta-\mathrm{Cn} \mathrm{A}$. Le dispositif expérimental, le comportement des laits en fabrication fromagère ainsi que les rendements obtenus et la composition des fromages frais sont décrits dans un premier article (Delacroix-Buchet et Marie, 1994). Les résultats font apparaître des différences importantes dans l'aptitude à la transformation fromagère des laits $\beta-\mathrm{Cn} C$ et $\beta-C n A$, au moment de la fabrication. Nous avons également suivi le déroulement de la protéolyse lors de l'affinage des minifromages et son incidence sur la texture et la flaveur des produits affinés. Les spécificités d'hydrolyse du variant $C$ de la caséine $\beta$ pouvaient, en effet, avoir des répercussions, lors de l'affinage d'un fromage comme le beaufort. $\mathrm{Ce}$ sont les résultats de ce suivi que nous présentons dans le présent article. Rappelons que l'objectif des essais est de caractériser au mieux les particularités du variant $C$ rare de la $\beta$-Cn par rapport à un témoin fréquent dans la plupart des races bovines (le variant $A$, englobant indifféremment le variant $A^{1}$ et le variant $A^{2}$ ) au niveau de la physico-chimie, de la rhéologie et de l'analyse sensorielle des fromages fabriqués à partir de ces laits.

\section{MATÉRIEL ET MÉTHODES}

Le protocole expérimental de fabrication des fromages est décrit en détail par Delacroix-Buchet et Marie (1994). Les dosages et mesures portent sur les paramètres permettant de suivre la protéolyse et de cerner l'incidence du variant $\mathrm{C}$ de la $\beta$-Cn sur la qualité des fromages. Le fromage a été analysé aux 3 stades d'affinage : $24 \mathrm{~h}$ (avant saumurage), 2 semaines et 8 semaines d'affinage.

\section{Analyses chimiques}

Toutes les analyses chimiques sont réalisées en double sur chaque échantillon.

\section{Fractions azotées}

Le dosage se fait par la méthode Kjeldhal automatisée (Vapodest 6, société Gerhardt, OSI, Paris, France) de l'azote des fractions soluble à $\mathrm{pH} 4,6$ (NS) et soluble dans l'acide phosphotungstique (NSAPT) des fromages âgés de $24 \mathrm{~h}$, 2 semaines et 8 semaines préparées selon la technique de Gripon et al (1975).

\section{Caséines}

L'analyse quantitative des caséines des fromages et de leurs produits de dégradation dans les fromages $\left(\alpha_{s 1}, \beta, \alpha_{s 1-1}, \gamma_{1}, \gamma_{2}, \gamma_{3}\right)$ est effectuée après séparation électrophorétique et lecture densitométrique (lecteur Vernon) de plaques d'électrophorèse selon la technique décrite pour les caséines des laits (Delacroix-Buchet et Marie, 1994). La caséine entière est préparée par dissolution dans $5 \mathrm{ml}$ d'urée $9 \mathrm{~mol} / \mathrm{l}$ contenant $1 \%$ de ß-mercaptoéthanol, de $0,3 \mathrm{~g}$ du précipité à $\mathrm{pH} 4,6$, récupéré lors de la préparation du NS.

\section{Peptides}

On effectue une séparation des constituants de la fraction NS des fromages âgés de 8 semaines par chromatographie liquide haute performance (HPLC) sur une colonne Delta Pak C18 $\left(300^{\circ} \mathrm{A}\right.$, $3,9 \times 150 \mathrm{~mm}, 5 \mu \mathrm{m})$ à l'aide d'une chaîne Waters (Milford, États-Unis) composée de 2 pompes haute pression (modèles 501 et 510 ), d'un injecteur automatique Wisp 712, d'un détecteur UV 484 , d'un logiciel de pilotage-acquisition de données Baseline 810. La colonne est thermostatée à $40^{\circ} \mathrm{C}$ grâce à un bain-marie à circulation d'eau. La séparation est effectuée en $30 \mathrm{~min}$ avec un gradient linéaire ( 0 à $70 \%$ de tampon $\mathrm{B}$ ) de 2 tampons à $\mathrm{pH} 7$ (tampon $\mathrm{A}=$ tampon phosphate $0,05 \mathrm{~mol} / \mathrm{l}$, tampon $\mathrm{B}=$ tampon $\mathrm{A} 40 \%$ + acétonitrile $60 \%, v / v)$ à un débit de $1 \mathrm{ml} / \mathrm{min}$. L'échantillon est préparé à partir d' $1 \mathrm{ml}$ de la solution de NS à laquelle on ajoute $1 \mathrm{ml}$ de TCA $4 \%$. Après centrifugation ( $10000 \mathrm{~g}, 3 \mathrm{~min}$ ), $400 \mu \mathrm{l}$ de surnageant sont filtrés à $0,45 \mu \mathrm{m}$ puis injectés auto- 
matiquement en tête de colonne. La détection des peptides se fait à $214 \mathrm{~nm}$.

\section{Plasmine-plasminogène}

Le dosage de l'activité de la plasmine dans le lait est effectué par la technique de Le Bars et Gripon (1989) qui consiste à quantifier par HPLC la libération d'un peptide provenant de l'hydrolyse par la plasmine de $\mathrm{I}^{\prime} \alpha_{\mathrm{s2}}-\mathrm{Cn}$ (fragment 1-24). Le lait à analyser est incubé $4 \mathrm{~h}$ à $37^{\circ} \mathrm{C}$ et le peptide est dosé avant et après incubation. La séparation des peptides est réalisée en $16 \mathrm{~min}$ avec la chaîne Waters précédemment décrite, sur une colonne Nucléosil C18 (5 um, 4,5 x $250 \mathrm{~mm}$ ), thermostatée à $37^{\circ} \mathrm{C}$, avec un gradient binaire (20 à $40 \%$ de $B$ en 10 min puis 40 à $100 \%$ de $B$ en $1 \mathrm{~min}$ et plateau à $100 \%$ de $B$ pendant $5 \mathrm{~min}$ ) de solvants à $\mathrm{pH} 2,2$ (solvant aqueux $\mathrm{A}=0,12 \%$ de TFA (acide trifluoroacétique) et solvant organique $B=0,10 \%$ TFA $+60 \%$ acétonitrile $v / v$ ). Le dosage immunochimique des quantités globales de plasmine et de plasminogène a été effectué par la technique Elisa compétitive mise au point par Collin et al (1990) sur le filtrat servant à la détermination de l'azote non caséinique (NCN) des laits préparé comme décrit par DelacroixBuchet et Marie (1994).

\section{Microstructure et rhéologie}

\section{Microstructure des fromages}

Une étude de la microstructure et de la compacité des fromages a été réalisée sur 2 fromages (un de type $\beta$-Cn A et un de type $\beta-\mathrm{Cn} C$ ) de la dernière série de fabrications et âgés de 48 h, 2 semaines et 8 semaines par microscopie électronique à balayage sur des sections délipidées d'échantillons secs. La préparation des échantillons consiste en une fixation de petits cubes de fromage (1 mm de côté) par le glutaraldéhyde à $2,5 \%$ dans un tampon cacodylate $0,1 \mathrm{~mol} / \mathrm{l}$ à $\mathrm{pH} 7,2$ pendant une durée de $48 \mathrm{~h}$ à $4^{\circ} \mathrm{C}$, suivie de rinçages en tampon, d'une deuxième fixation en $\mathrm{OsO}_{4} 1 \%$ dans le même tampon pendant $15 \mathrm{~h}$ à $4^{\circ} \mathrm{C}$, et de lavages. Les échantillons sont ensuite déshydratés par lalcool éthylique. Ils sont séchés par la méthode au point critique (éthanol- $\mathrm{CO}_{2}$ liquide) puis métallisés à l'or-paladium (couche de $18 \mathrm{~nm}$ ). L'observation est faite avec un microscope Hitachi S- 450 sous une tension de $15 \mathrm{kV}$.

\section{Texture des fromages}

Des tests de compression simple uniaxiale ont été effectués sur les fromages âgés de 8 semaines, à l'aide d'une machine Instron SA modèle 1102 (Buc, France). Une carotte cylindrique de $24 \mathrm{~mm}$ de diamètre a été découpée à l'aide d'un emporte-pièce, au centre des fromages déparaffinés, sur toute la hauteur du fromage. La hauteur du cylindre a été ajustée à $20 \mathrm{~mm}$ par une découpe symétrique des deux bases du cylindre. L'éprouvette de fromage a été maintenue $1 \mathrm{~h}$ à $20^{\circ} \mathrm{C}$ à l'abri de toute dessiccation dans un pèse-filtre, jusqu'au moment du test. Puis, celleci a été comprimée à vitesse constante $(25$ $\mathrm{mm} / \mathrm{min}$ ) jusqu'à une déformation de $80 \%$ de sa hauteur initiale dans un local climatisé à $20^{\circ} \mathrm{C}$. A partir des courbes de compression, on a déterminé 4 valeurs rhéologiques, le module d'élasticité dit module de Young qui est la pente de la courbe à l'origine $(\mathrm{PO})$ déterminée dans la partie linéaire de la courbe et qui représente l'élasticité du fromage, la contrainte à la rupture (CR), considérée comme représentant la dureté de la pấte, la déformation d'après Cauchy au point de rupture (DR) $(\%)$ qui s'assimile à l'état de cohésion de la pâte et la contrainte maximale (CM) qui est intraduisible en terme de structure, celle du fromage étant détruite.

\section{Analyse sensorielle}

Les fromages ont été dégustés à 8 semaines d'affinage, dans un local spécialement prévu à cet effet (AFNOR, 1972). Le jury était composé de 6 à 8 dégustateurs entraînés (Vassal, 1984). A chaque épreuve correspondait une série de fabrications et les comparaisons portaient directement sur les 2 ou 3 fromages fabriqués un même jour. Des portions de fromages de 15 à $20 \mathrm{~g}$ étaient présentées codées au jury dans un ordre aléatoire et différent pour chaque dégustateur. Le jury disposait de pain et d'eau pour se rincer la bouche entre chaque échantillon. Les analyses sensorielles étaient de type profil analytiquedescriptif. L'épreuve sensorielle comportait 2 étapes. Dans un premier temps, il était demandé aux membres du jury de décrire les sensations visuelles (aspect), tactiles (par écrasement entre les doigts) et olfactives perçues lors de l'examen des échantillons de fromage. Dans un second temps, chaque membre du jury devait noter, sur une échelle structurée à 5 niveaux (note de 0 à 4), 
une liste de descripteurs, préalablement retenus, de texture (3), de flaveur (6), ainsi que l'intensité globale d'odeur et de flaveur et évaluer la qualité du produit. Les 2 fromages de la fabrication $n^{\circ} 3$, qui présentaient des contaminations, n'ont pas été dégustés.

\section{Analyses statistiques}

Les analyses statistiques utilisées sont décrites dans l'article précédent (Delacroix-Buchet et Marie, 1994, Lait 74, 4, 343-360). Pour l'analyse sensorielle, chaque fromage s'est vu attribuer une note correspondant à la moyenne des notes du jury. Chaque moyenne est intégrée dans l'étude statistique au même titre qu'une valeur analytique. Pour certaines variables qui, contrairement à notre attente, n'étaient pas discriminantes, nous avons réalisé une analyse de covariance. Cette technique associe une analyse de variance et une régression, et permet d'éliminer les effets des covariables pour mieux analyser le facteur contrôlé du dispositif expérimental.

Le traitement statistique des résultats a été effectué à l'aide du logiciel MICROSTAT élaboré en 1984 par l'ITCF (Boigneville, France) à l'exception des analyses de variance à 3 facteurs ("génotype" et "jour de fabrication" en effets fixes et "vache" en effet aléatoire) et de variancecovariance réalisées avec le logiciel SAS (SAS Institute, Inc, Cary, NC, États-Unis) selon la procédure GLM.

\section{RÉSULTATS}

\section{Protéolyse}

\section{Protéolyse primaire}

La protéolyse primaire correspond aux premières coupures des caséines $\alpha_{s}$ et $\beta$. Leur dégradation peut être suivie par l'analyse des électrophorèses sur gel PAGE (fig 1). Les bandes électrophorétiques correspondant à la $\beta$-Cn et à la fraction $\gamma_{1}$, issue de l'hydrolyse de la $\beta$-Cn par la plasmine (fragment 29-209), dans les fromages, ont des

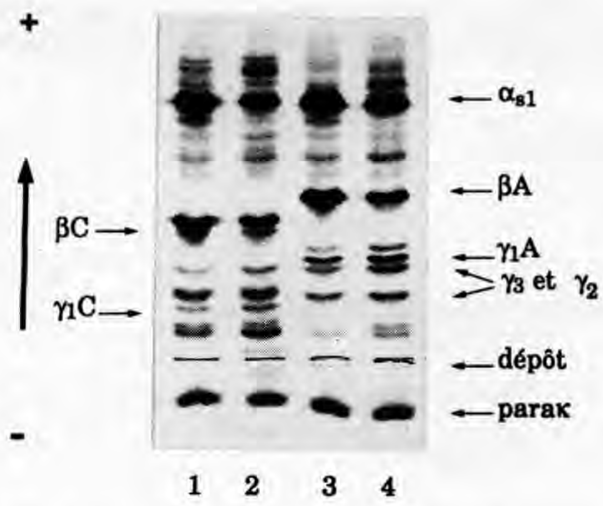

Fig 1. Analyse électrophorétique (PAGE pH 8,6) de 2 échantillons de fromages à 2 stades successifs d'affinage. 1) $\beta-C n C$ à 2 semaines ; 2) $\beta$-Cn $C$ à 8 semaines ; 3$) \beta-C n$ A à 2 semaines ; 4) $\beta-\mathrm{Cn} A$ à 8 semaines.

Electrophoretic analysis (PAGE pH 8.6) of cheese samples at two successive stages of ripening process (effect of $\beta$-casein variants). Lane 1) $\beta$ $\mathrm{Cn} C$ at 2 weeks; Lane 2) $\beta$-Cn $C$ at 8 weeks; Lane 3) $\beta$-Cn A at 2 weeks; Lane 4) $\beta-C n A$ at 8 weeks.

mobilités différentes selon le variant génétique de la $\beta-C n$.

Aucune différence significative n'apparaît dans les proportions résiduelles de caséine $\alpha_{s 1}(27,3$ contre $26,7 \%$ de la caséine totale) et de caséine $\beta(22,7$ contre $20,0 \%$ de la caséine totale) respectivement entre génotypes $C$ et $A$, après 8 semaines d'affinage des fromages.

On met en revanche en évidence une différence hautement significative dans la proportion de fraction $\gamma_{1}$ entre les variants $A$ et $C$ de la $\beta-C n(7,9$ contre $3,7 \%$ de la caséine totale ; $P<0,01$ ) (tableau I). La para $\kappa-C n$, associée à la $\beta-C n$ A est aussi présente en plus grande proportion dans la caséine des fromages âgés de 8 semaines (7,4 contre $5,7 \% ; P=0,07)$. Au contraire, la fraction $\gamma_{2}$ a tendance à être en proportion plus importante dans les minifromages $\beta$ Cn C $(6,5$ contre $4,9 \%$ de la caséine totale ; 
Tableau I. Protéolyse des fromages en cours d'affinage (effet des variants de la caséine $\beta$ ). Proteolysis of cheeses during ripening (effect of $\beta$-casein variants).

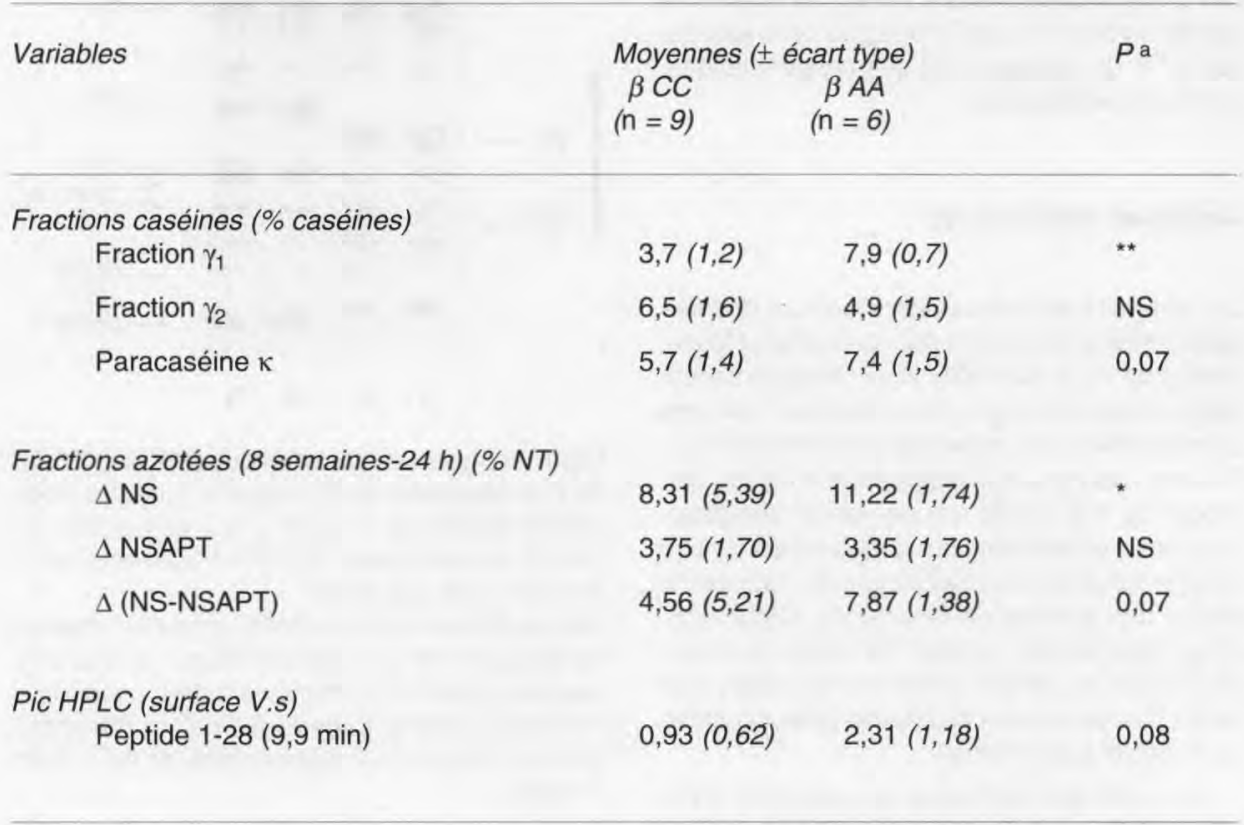

a $P$ : seuil de probabilité suivant le modèle à 3 facteurs (effets "génotype" et "jour de fabrication", effet "vache" en aléatoire). NS: $P>0,10 ;{ }^{\star} P<0,05 ;{ }^{\star \star} P<0,01$.

$P=0,15)$. Peu de différences apparaissent pour les autres fractions caséiniques présentes dans le fromage.

\section{Protéolyse secondaire}

La protéolyse secondaire a été mesurée globalement par les teneurs en azote de la fraction azotée soluble à $\mathrm{pH} 4,6$ (NS) qui représente les polypeptides et les acides aminés, et la fraction soluble dans l'acide phosphotungstique (NSAPT) qui regroupe les di- et tripeptides et les acides aminés, à l'exclusion des acides di-aminés. Une étude plus approfondie a été réalisée en examinant les profils peptidiques obtenus en chromatographie HPLC.
Au cours de l'affinage, l'azote total est plus vite dégradé dans les fromages $\beta$ $\mathrm{Cn} A$ que dans les fromages $\beta-\mathrm{Cn} C$ (tableau I). Les fromages $\beta$-Cn A présentent des rapports NS/NT plus élevés que les fromages $\beta$-Cn C. L'écart entre les 2 génotypes augmente en cours d'affinage, et si la différence n'est jamais significative pour un stade d'affinage donné, l'augmentation de NS entre le début ( $24 \mathrm{~h}$ ) et la fin d'affinage ( 8 semaines) est significativement plus élevée pour les fromages $\beta$ Cn A $(11,2$ contre $8,3 \%$ NT ; $P<0,05)$. En revanche, les différences entre les 2 génotypes pour la teneur en NSAPT ne sont pas importantes. La protéolyse secondaire semble donc globalement similaire pour les deux types de fromage. La différence 
de NS entre les 2 génotypes porte essentiellement sur les gros peptides (ce que traduisent les valeurs de NS-NSAPT), donc sur les produits issus de la dégradation primaire des caséines.

L'intégration des pics obtenus sur les profils peptidiques de chaque fromage a mis en évidence des différences dans les surfaces intégrées, en particulier pour un temps de rétention de 9,90 $\mathrm{min}$. Les fromages âgés de 8 semaines de type $\beta-C n A$ présentent une surface pour ce pic de 2 à 2,5 fois plus importante que les fromages $\beta-C n C(0,93$ contre 2,31 V.s ; $P=0,08)$. On peut ajouter qu'une forêt de pics apparaît entre 27 et 30 min de rétention dans le profil $\beta-C n A$, alors qu'elle est peu marquée dans le profil peptidique des fromages $\beta$ Cn C (fig 2). Les molécules correspondant à ces pics sont malheureusement difficilement identifiables du fait de la complexité de tels profils. Tous les produits d'hydro- lyse des caséines, solubles dans le TCA $2 \%$ et présents dans les fromages, se retrouvent dans la fraction analysée. Cependant, on note une corrélation négative entre le pic à 9,90 min de rétention et la proportion de $\beta$-Cn $(r=-0,659 ; P<0,01)$ et une corrélation positive entre ce même pic et la fraction $\gamma_{1}(r=0,751 ; P<0,001)$ (fig 3).

Aucune différence significative n'est relevée, entre les génotypes $C$ et $A$, dans les teneurs en plasmine-plasminogène des laits (respectivement 3,2 contre $2,9 \mathrm{mg} / \mathrm{ml}$ ) ou dans l'activité de la plasmine, représentée par la surface du pic HPLC $(0,24$ contre 0,30 V.s).

\section{Microstructure et rhéologie}

Nous décrirons d'abord la microstructure du fromage, c'est-à-dire l'arrangement spa-

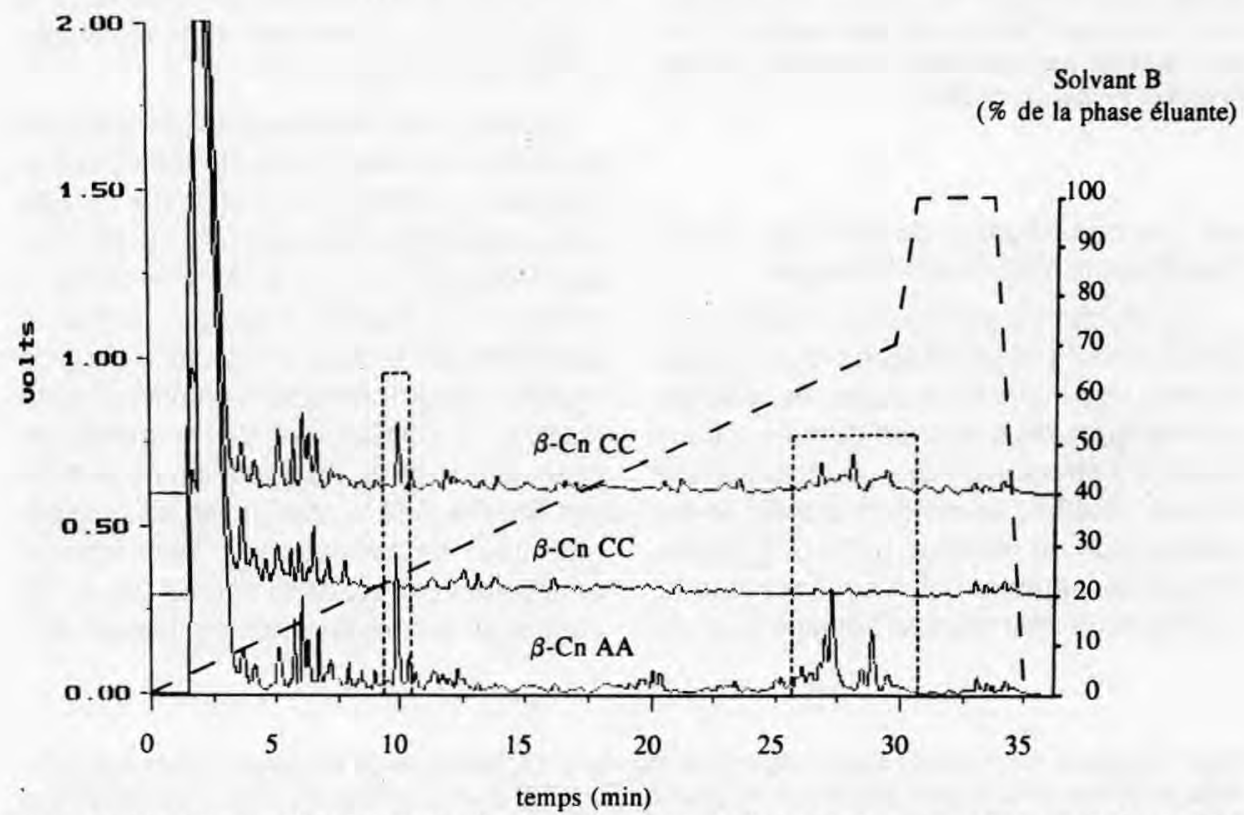

Fig 2. Profils d'élution HPLC de la fraction azotée soluble à $\mathrm{pH} 4,6$ de fromages de types $\beta$-Cn $\mathrm{A}$ et $\beta$ $\mathrm{Cn} C$ à 8 semaines d'affinage (conditions d'analyses décrites dans le texte).

$H P L C$ elution profiles of soluble nitrogen fractions at $p H 4$.6, from $\beta$-Cn A and $\beta$-Cn C types of cheese at 8 weeks of ripening (analysis conditions described in the text). 


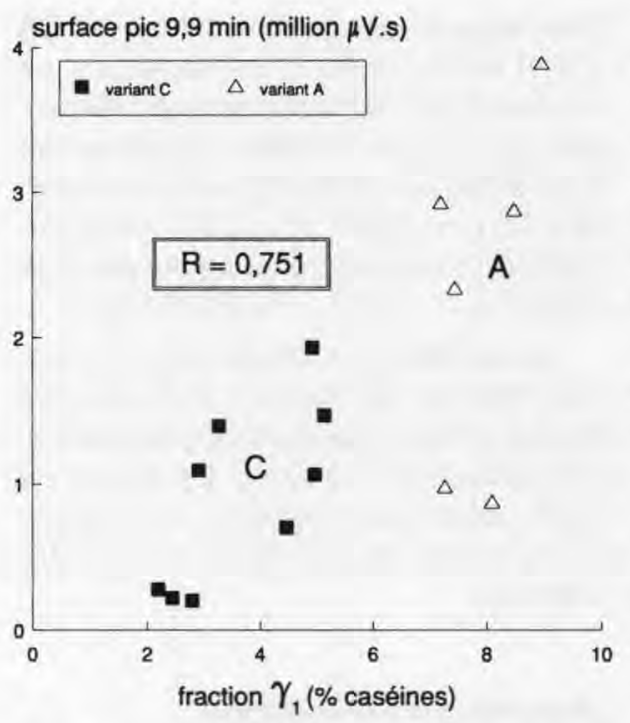

Fig 3. Relation entre l'aire du pic HPLC élué à 9,90 min et la proportion de fraction $\gamma_{1}$ résiduelle à 8 semaines d'affinage des fromages (effet des variants de la caséine $\beta$ ).

Relationship between the surface of the HPLC peak eluted at $9.90 \mathrm{~min}$ and the proportion of $\gamma 1$ residual fraction at 8 weeks of cheese ripening (effect of $\beta$-casein variants).

tial des constituants du fromage, avant d'analyser la texture des fromages.

- La structure d'un fromage à pâte pressée cuite se présente après le pressage comme une matrice compacte et continue de filaments de caséines gonflés d'eau, enserrant étroitement des gouttelettes lipidiques. À $48 \mathrm{~h}$, la matrice protéique de l'échantillon de fromage $\beta-\mathrm{Cn} C$, quoique compacte, semble moins continue que la matrice de l'échantillon de fromage $\beta-C n A$.
Dans la pâte, les logements des globules gras de l'échantillon $\beta$-Cn $C$ sont moins bien délimités que ceux de l'échantillon $\beta-C n A$, ils sont moins sphériques et de forme irrégulière (photos $A$ et $B$, grandissement $500 \mathrm{X}$; fig 4). À 2 semaines, la différence de structure des pâtes semble encore plus nette qu'à $48 \mathrm{~h}$ (photos $\mathrm{C}$ et $\mathrm{D}$, grandissement $2000 \mathrm{X}$; fig 4). À 8 semaines, la différence de structure est, en revanche, beaucoup moins apparente. La caséine présente une structure plus lisse, plus affinée et les logements des globules gras sphériques sont bien délimités pour les 2 types de fromage (photos $D$ et $F$, grandissement $2000 \mathrm{X}$; fig 4). À ce grandissement, la décomposition de la caséine n'est pas visible.

Les paramètres mesurés lors du test de compression, sur les fromages âgés de 8 semaines, sont corrélés entre eux. La contrainte à la rupture est liée positivement $(r=0,728 ; P<0,01)$ à la déformation à la rupture, ainsi qu'à la contrainte maximale $(r=0,701 ; P<0,01)$.

La contrainte maximale est étroitement corrélée à la teneur en azote total des fromages ( $r=0,898 ; P<0,001)$ (fig 5). Elle est aussi négativement corrélée au gras/sec des fromages $(r=-0,615 ; P<0,05)$. II existe une liaison négative entre la contrainte à la rupture et l'humidité par $\mathrm{g}$ de caséine, rapport considéré comme un estimateur du gonflement des micelles de caséine du fromage. La relation ne semble pas linéaire mais le nombre de points expérimentaux ne permet pas d'envisager un ajustement correct de la courbe (fig 6). La contrainte à la rupture est également cor-

Fig 4. Étude par microscopie électronique de la structure d'échantillons de fromages à 3 stades d'affinage (effet des variants de la caséine $\beta$ ). A) $\beta-C n A$ à $48 \mathrm{~h}$; B) $\beta-C n C$ à $48 \mathrm{~h}$; C) $\beta-C n$ A à 2 semaines ; D) $\beta$-Cn $C$ à 2 semaines ; E) $\beta$-Cn A à 8 semaines et F) $\beta$-Cn $C$ à 8 semaines.

Electron microscopy study of cheese samples at 3 ripening stages (effect of $\beta$-casein variants). A) $\beta$ $C n A$ at $48 h$; B) $\beta$-Cn $C$ at $48 h$;C) $\beta$-Cn A at 2 weeks; D) $\beta$-Cn $C$ at 2 weeks; E) $\beta$-Cn A at 8 weeks and F) $\beta$-Cn $C$ at 8 weeks. 

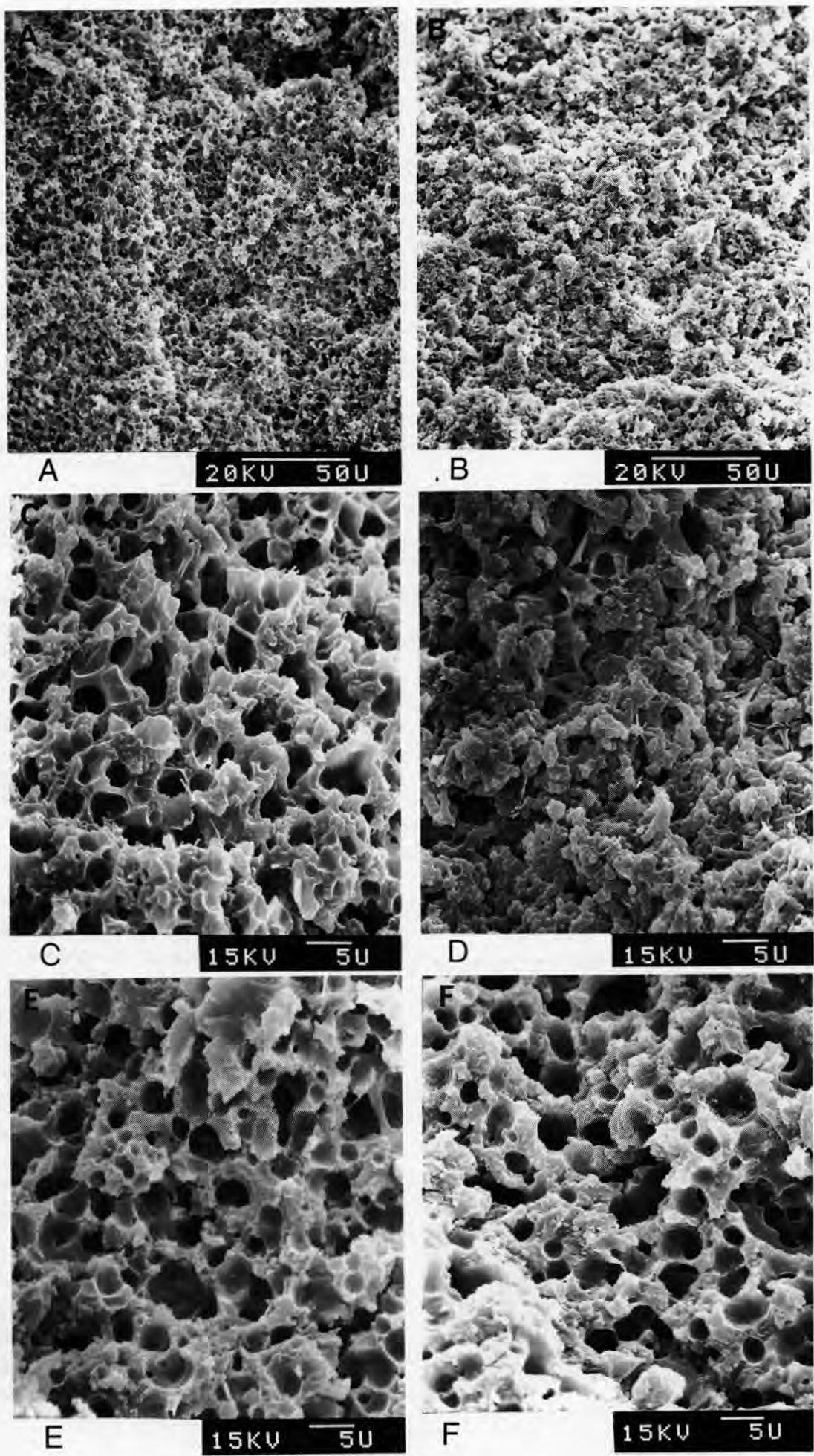


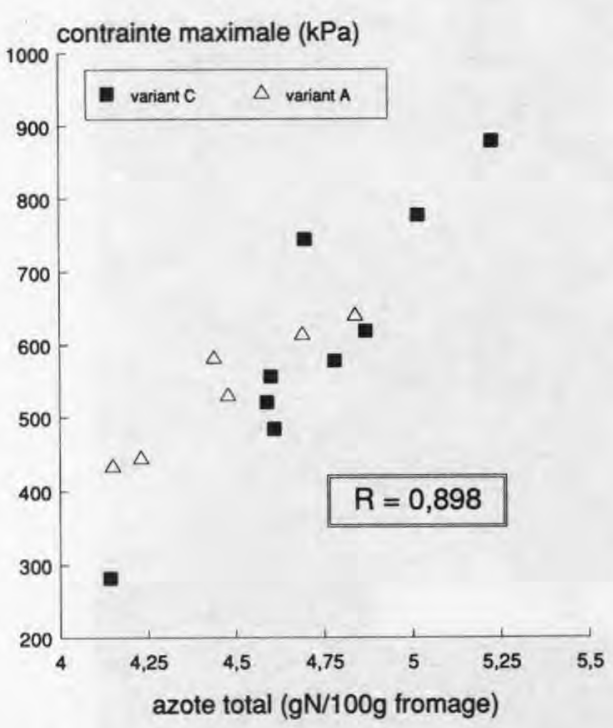

Fig 5. Relation entre la teneur en azote total et la contrainte maximale des fromages à 8 semaines d'affinage (conditions du test de compression décrites dans le texte) (effet des variants de la caséine $\beta$ ).

Relationship between the nitrogen content and the maximum stress of cheese samples at 8 weeks of ripening (conditions of the compression test described in the text) (effect of $\beta$-casein variants).

rélée à la proportion d'azote soluble dans l'azote total $(r=-0,627 ; P<0,05)$.

L'analyse de variance sur les données rhéologiques brutes n'apporte que peu de renseignements. On remarque que la courbe de compression des fromages $\beta$ $\mathrm{Cn} \mathrm{C}$ semble avoir une pente à l'origine plus importante, traduisant une élasticité moindre que celle des fromages $\beta$-Cn A (46,2 contre $38,2 ; P=0,13)$. En moyenne, la contrainte à la rupture est de $235 \mathrm{kPa}$, quel que soit le génotype. La déformation à la rupture et par conséquent la cohésion de la pâte est un peu plus élevée pour les fromages $\beta$ $\mathrm{Cn} A$ que pour les fromages $\beta$ - $\mathrm{Cn} C(53,0$ contre $49,7 \%$ ), mais la différence n'est pas significative.

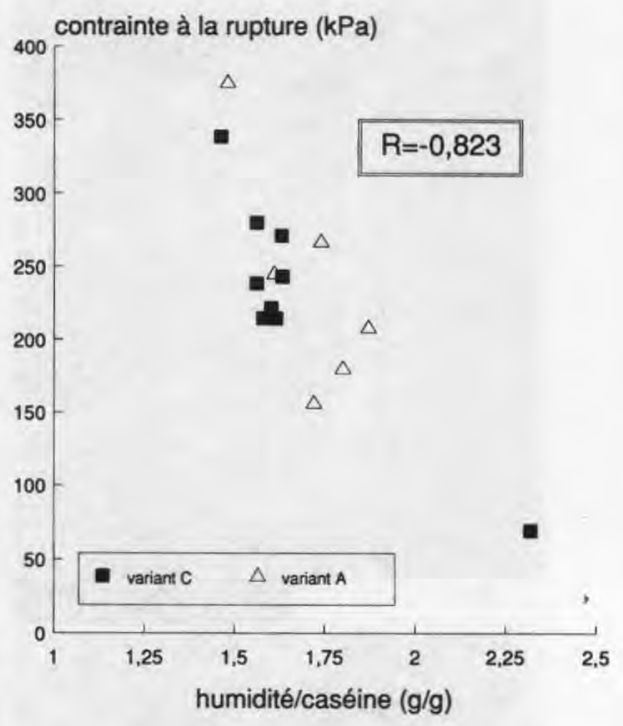

Fig 6. Relation entre le rapport humidité/caséine et la contrainte à la rupture des fromages à 8 semaines d'affinage (conditions du test de compression décrites dans le texte) (effet des variants de la caséine $\beta$ ).

Relationship between the ratio moisture/casein and the fracture stress of cheese samples at 8 weeks of ripening (conditions of the compression test described in the text) (effect of $\beta$-casein variants).

Dans un deuxième temps, nous avons réalisé des analyses de variance-covariance pour améliorer la précision du modèle d'analyse de variance. Avec le modèle à 3 facteurs (génotype, jour de fabrication et vache), la contrainte à la rupture présente un $r^{2}$ de $0,49, r^{2}$ qui passe à 0,99 lorsque l'on met en covariable l'azote total des fromages à 8 semaines. L'utilisation de cette covariable (qui apporte une information très significative sur la contrainte à la rupture ; $P=$ 0,01 ) permet de mettre en évidence une différence significative entre les 2 types de fromages $(P=0,02)$. La précision du modèle appliqué à la contrainte maximale est aussi améliorée lorsque l'on prend en compte la teneur en azote total des fromages. Bien qu'apportant une information non négli- 
geable $(P=0,05)$, l'introduction d'une covariable ne permet pas de conclure à un effet significatif du génotype sur la contrainte maximale (écart moyen de $12 \%$ entre les fromages $\beta-C n C$ et $\beta-C n A$ ). On peut cependant globalement admettre que les laits des animaux $\beta$-Cn $\mathrm{C}$ donnent des fromages plus fermes.

\section{Analyse sensorielle}

Les termes générés pour décrire les fromages montrent qu'ils ont globalement le même aspect visuel : la pâte est compacte, sans ouverture, mais qu'il existe des différences dans la texture des fromages (appréciée entre les doigts) : la pâte des fromages de type $\beta-C n$ A est souple, alors que celle des fromages $\beta-C n C$ est sableuse, voire friable. L'intensité d'odeur est voisine pour les deux types de fromages (de l'ordre de $1,5 / 4)$ mais l'odeur des fromages $\beta$-Cn $A$ est qualifiée de douce alors que celle des fromages $\beta-C n C$ est plutôt qualifiée d'acide ou de caillé frais.

L'analyse statistique des paramètres de dégustation a révélé 3 paramètres permettant de différencier les fromages (fig 7). Les minifromages de type $\beta$-Cn $\mathrm{C}$ ont un goût noté plus souvent piquant $(0,94 / 4$ contre $0,39 / 4 ; P=0,13$ ), à tendance plus acide $(1,13 / 4$ contre $0,71 / 4)$ associé à une pâte plus dure $(2,87 / 4$ contre $2,37 / 4 ; P=0,23)$. Les fromages fabriqués à partir de laits $\beta$ $\mathrm{Cn} \mathrm{A}$ ont une saveur plus douce $(1,61 / 4$ contre $1,22 / 4 ; P=0,24$ ) voire un goût plus fruité $(1,60 / 4$ contre $1,34 / 4)$. Le goût piquant est négativement corrélé à la saveur douce $(r=-0,640 ; P<0,05)$ et positivement corrélé à la saveur acide $(r=0,652 ; P<0,05)$. Les fromages les plus savoureux sont un peu plus fruités $(r=0,524)$. Les fromages ne sont ni amers (note moyenne : 0,45/4), ni rances (note moyenne : $0,25 / 4$ ). a.

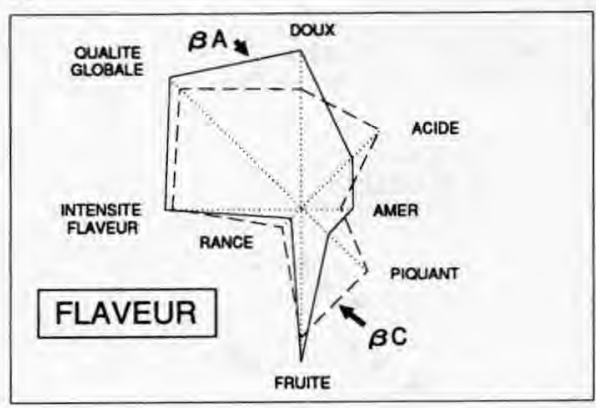

b.

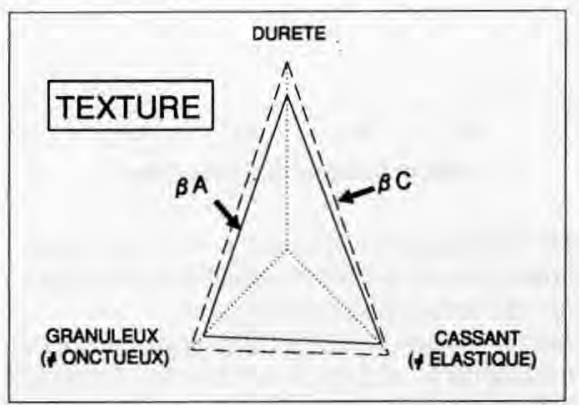

Fig 7. Profils sensoriels comparatifs des fromages de type $\beta$-Cn A et $\beta$-Cn C à 8 semaines d'affinage. a) flaveur; $b$ ) texture.

Sensory comparative profiles of $\beta-C n A$ and $\beta$ Cn $C$ types of cheese at 8 weeks of ripening. a) flavour ; b) texture.

Le goût piquant est inversement lié à la proportion de fraction $\gamma_{1}$ et de para $\kappa-C n$ dans la caséine ainsi qu'à l'aire du pic HPLC élué à $9,90 \mathrm{~min}$ (respectivement $r=-0,610$; $r=-0,670$ et $r=-0,678 ; P<0,05)$. Les fromages à la saveur la plus douce ont la teneur en sel la plus faible $(r=-0,737$; $P<0,01$ ) et ils sont fabriqués à partir de laits plus riches en calcium colloïdal $(r=0,551 ; P$ $<0,05$ ) (fig 8 ). Le goût fruité est négativement corrélé à la proportion de fraction $\gamma_{2}$ dans la caséine $(r=-0,620 ; P<0,05)$.

Les fromages les plus granuleux (en bouche) sont les moins élastiques ( $r=$ $-0,608 ; P<0,05)$. Le caractère dur des fromages est bien corrélé à la contrainte à la 


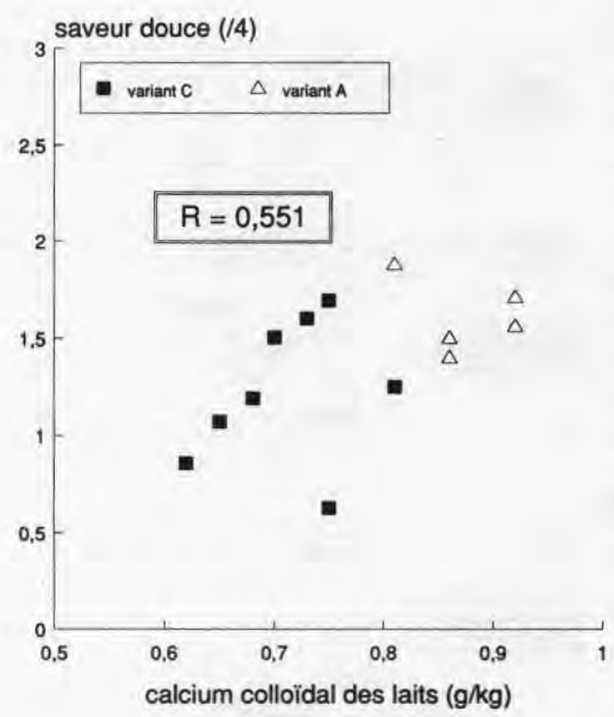

Fig 8. Relation entre la teneur en calcium colloïdal des laits et la saveur douce des fromages (effet des variants de la caséine $\beta$ ).

Relationship between the colloidal calcium content of milk samples and the sweet savour of cheese (effect of $\beta$-casein variants).

rupture mesurée lors du test de compression $(r=0,574 ; P<0,05)$ mais surtout à la contrainte maximale $(r=0,702 ; P<0,01)$. Le caractère élastique est quant à lui bien corrélé à la déformation à la rupture ( $r=$ $0,772 ; P<0,01)$.

La note d'évaluation globale des minifromages n'est pas significativement différente pour les fromages de type $\beta-C n C$ ou $\beta$-Cn $A$ (respectivement $1,73 / 4$ et $1,90 / 4$ ). Les fromages les plus appréciés sont les moins acides $(r=-0,759 ; P<0,01)$, les plus élastiques $(r=0,759 ; P<0,01)$ et ont l'intensité de flaveur la plus forte $(r=0,574 ; P<0,05)$.

\section{DISCUSSION}

Les minifromages expérimentaux sont globalement moins protéolysés que des beau- forts affinés. Les rapports NS/NT dans les minifromages âgés de 8 semaines sont en moyenne beaucoup plus faibles (15 à $17 \%$ ) que dans les fromages de Beaufort affinés (25 à 32\% de l'azote total) (Chamba et al, 1994). Bien que la température d'affinage soit de $12^{\circ} \mathrm{C}$ pour les minifromages (au lieu de 8 a $10^{\circ} \mathrm{C}$ pour les beauforts traditionnels), la durée d'affinage de 2 mois (au lieu de 4 ou 6 mois) ne permet pas d'atteindre un stade identique de protéolyse. De plus, Kerjean et al (1987) ont déjà observé dans des fromages miniatures une protéolyse limitée, sans en donner d'explication.

Le degré de protéolyse des minifromages est cependant suffisant pour mettre en évidence des différences quantitatives et qualitatives dans l'hydrolyse des caséines avec les laits des deux génotypes. Les différences d'hydrolyse de la $\beta-C n$ selon le génotype ne s'expliquent pas par des taux de plasmine différents selon le génotype. En effet, les dosages de plasmine effectués sur les laits montrent qu'il n'y a pas, en moyenne, de différence significative entre les génotypes. Les paramètres les plus importants de variation du taux de plasmine sont la saison et le stade de lactation de l'animal (Barry et Donnelly, 1980 ; Schaar, 1985 ; Benslimane et al, 1990). Or les animaux ont été prélevés sur une courte période et à des stades voisins de lactation $\left(92^{\mathrm{e}}\right.$ jour pour les $\mathrm{C}$ et $103^{\circ}$ jour pour les $\left.\mathrm{A}\right)$. En fabrication de pâte cuite pressée, il a été mesuré que l'activité de la plasmine du fromage est environ 10 fois plus importante que celle du lait, ce qui correspond au facteur de concentration du lait en fromage. Ce résultat a été obtenu en ajoutant artificiellement des doses croissantes de plasmine active dans un même lait destiné à des fabrications simultanées d'emmental dans des minicuves (Delacroix-Buchet, résultats non publiés). Par conséquent, l'absence de différences constatées dans les taux moyens de plasmine des 2 types génétiques de laits étudiés doit être également vraie dans les fro- 
mages. D'autre part, la teneur en $\mathrm{NaCl}$ des fromages varie peu (Delacroix-Buchet et Marie, 1994). En moyenne, cette teneur est voisine de $1,4 \%$ dans le fromage, soit $3,5 \%$ dans l'humidité du fromage, zone dans laquelle l'activité de la plasmine est peu affectée par des variations de la teneur en sel des fromages (Delacroix-Buchet et Trossat, 1991). Les différences de teneur en NS entre les 2 types de fromages ne peuvent pas être liées non plus à des différences dans l'activité des levains car la quantité de NSAPT est comparable pour les 2 génotypes. Par ailleurs, les levains qui sont responsables de la production de NSAPT (Desmazeaud et al, 1976) sont identiques pour les 2 types de fromages.

Les observations faites par Papoff et al (1994) sur l'hydrolyse par la plasmine de caséines purifiées $\beta$-Cn $A^{\dagger}$ et $\beta-C n C$ montrent que les résultats dans les minifromages sont comparables à ceux obtenus après une à deux heures d'hydrolyse de la $\beta$ Cn purifiée par la plasmine, dans des conditions optimales. II découle de ce qui précède que dans les fromages, les seules différences de protéolyse observées sont liées à des différences dans la cinétique d'hydrolyse par la plasmine des variants de la $\beta-C$. L'hydrolyse de la $\beta-C n C$, par la plasmine, est plus lente que celle de la $\beta$ $\mathrm{Cn} \mathrm{A}$ et les produits d'hydrolyse sont moins nombreux.

La migration électrophorétique plus lente de la $\beta-C n C$ et de la fraction $\gamma_{1} C$ provient de la déphosphorylation du résidu Ser 35 (Papoff et al, 1994). Corradini et Battistotti (1973) ont comparé des fromages fabriqués avec du lait d'une race voisine de la Simmental et du lait de Holstein-Frisonne, l'une possédant le variant $C$ de la $\beta-C n$, l'autre pas. Ils ont aussi observé, sans leur donner une interprétation génétique, des différences dans la mobilité électrophorétique (gel d'amidon) des produits d'hydrolyse de la $\beta-C n$ entre les 2 types de fromages. Les fromages de Beaufort fabriqués avec des

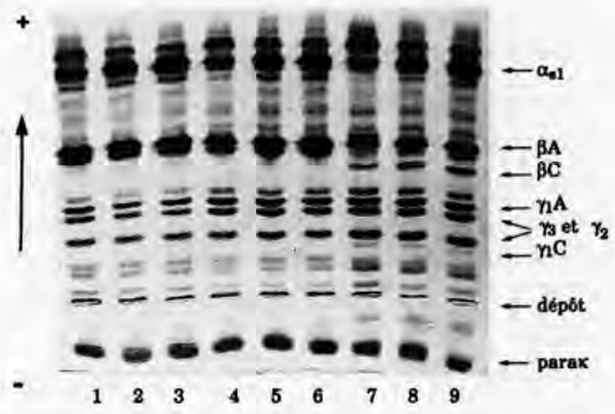

Fig 9. Séparation électrophorétique d'échantillons de fromages à pâte cuite pressée âgés de 50 j, fabriqués le même jour et affinés différemment. 1 , 2 et $3:$ emmentals ; 4,5 et $6:$ gruyères de Comté ; 7,8 et 9 : beauforts. Conditions d'affinage : 3 semaines à $11^{\circ} \mathrm{C}$ puis 1,4 et $7: 23^{\circ} \mathrm{C}$ et $80 \%$ d'humidité relative ; 2,5 et $8: 18^{\circ} \mathrm{C}$ et $96 \%$ d'humidité relative ; 3,6 et $9: 11^{\circ} \mathrm{C}$ et $96 \%$ d'humidité relative.

Electrophoretic patterns of samples of cooked curd type of cheese aged 50 days, made the same day and ripened under different conditions. Lanes 1, 2, 3: Emmental; lanes 4, 5, 6: Gruyère of Comté; lanes 7, 8, 9: Beaufort. Ripening conditions: 3 weeks at $11^{\circ} \mathrm{C}$ then lanes 1,4 and 7 : $23^{\circ} \mathrm{C}$ and $80 \%$ relative humidity; lanes 2,5 and 8 : $18^{\circ} \mathrm{C}$ and $96 \%$ relative humidity; lanes 3, 6 and 9 : $11^{\circ} \mathrm{C}$ and $96 \%$ relative humidity.

laits de mélange présentent, au niveau de la $\beta-C n$ et des fractions $\gamma$, un nombre plus important de bandes électrophorétiques. comparés à d'autres fromages á pâte cuite pressée (emmental ou gruyère de Comté) (fig 9, document ITG non publié). Cette observation est liée à la présence du variant $C$ de la $\beta-C n$ dans les caséines de fromage de Beaufort.

Le peptide élué à $9,90 \mathrm{~min}$ dans les profils HPLC peut être identifié au peptide 1-28 de la $\beta$-Cn d'après les profils peptidiques réalisés par Papoff et al (1994), dans les mêmes conditions chromatographiques. Dans ce cas, la corrélation entre la proportion de fraction $\gamma_{1}$ (fragment 29-209 de la $\beta-C n)$ dans la caséine et l'aire du pic HPLC 
à 9,90 min démontre la cohérence des résultats obtenus dans les fromages.

Le comportement différent de la fraction $\gamma_{1}$ par rapport aux fractions $\gamma_{2}$ et $\gamma_{3}$ est difficilement explicable. II a déjà été observé par Kaminogawa et Yamauchi (1972), dans une étude sur la plasmine et Collin et al (1987), dans une étude sur le gruyère de Comté.

La différence dans la proportion en para $\mathrm{-}-\mathrm{Cn}$, entre fromages des 2 types n'est, semble-t-il, que le reflet de la plus grande concentration en $\kappa-\mathrm{Cn}$ dans les laits de type $A$ à plus faible diamètre micellaire (Delacroix-Buchet et Marie, 1994).

Les différences dans la composition des micelles de caséines $\beta-C n A$ et $\beta-C n C$ et dans la protéolyse du réseau de caséines, au cours de l'affinage des 2 types de fromages, expliquent les différences de structure et de qualité des fromages.

Quand les pertes minérales dans le caillé sont faibles (cas des technologies à pâte cuite oủ l'acidification est limitée), l'identité des submicelles de caséine dans le fromage est pratiquement inchangée par rapport à celle du lait d'origine (Lawrence et al, 1983). L'aspect à $48 \mathrm{~h}$, de la pâte des fromages $\beta$-Cn C, observée au microscope électronique, est ainsi la conséquence d'une caséine moins ferme, de la présence d'une plus grande quantité d'eau, d'une quantité de calcium plus faible que dans les fromages $\beta-C n A$, entraînant une hydratation moindre de la matrice de caséine (Lawrence et al, 1987). À ce stade, la pâte des fromages $\beta-C n C$ présente des similitudes de structure avec celle des fromages à pâte molle plus acides et moins minéralisés (Rousseau, communication personelle). À 2 semaines, la différence plus nette de structure des pâtes $\beta$-Cn $A$ et $\beta$ - $C n C$ s'explique par l'affinage plus lent des fromages $\beta-\mathrm{Cn} C$. Pour les fromages à pâte cuite, la remontée de $\mathrm{pH}$ est faible pendant les 14 premiers jours, notamment pour les fromages $\beta$-Cn $C$ (Delacroix-Buchet et Marie, 1994). À ce stade, le $\mathrm{pH}$ moyen des fromages $\beta$-Cn $\mathrm{C}$ varie de 5,1 à 5,2 et celui des fromages $\beta$ $\mathrm{Cn} \mathrm{A}$ de 5,2 à 5,3. Or, entre $\mathrm{pH} 5,1$ et 5,3, les submicelles de caséine présentent de grandes différences dans leur taille et leurs caractéristiques, ce qui modifie leur capacité à fixer l'eau (Lawrence et al, 1987). Le gonflement des caséines dépend aussi directement de leur protéolyse puisque l'eau se fixe sur les groupes terminaux des produits d'hydrolyse comme par exemple, les groupes aminés ou les groupes carboxylés (Knoop et Buchheim, 1980). À 8 semaines, le degré de protéolyse et le gonflement de la matrice de caséine sont tels que l'on n'observe plus de différence dans l'aspect général de la pâte des fromages au grandissement utilisé.

La matrice de protéines détermine la consistance du fromage (Steffen, 1975; Chen et al, 1979; Walstra et Van Vliet, 1982). Ainsi, plus la pâte est riche en azote, plus la contrainte maximale devra être importante pour comprimer l'échantillon jusqu'à $20 \%$ de sa taille initiale. Rüegg et al (1980) ont montré qu'une forte concentration en matière grasse constitue un facteur de ramollissement du fromage car les globules gras interrompent la matrice de caséine (Walstra et Van Vliet, 1982). Ainsi, laugmentation du gras/sec des fromages provoque une diminution de la fermeté des fromages. Une humidité plus forte de la pâte du fromage réduirait la friction entre les micelles de caséines (Lawrence et al, 1982 ) et aurait un effet ramollissant. A l'opposé, une réduction de cette humidité (diminution de l'eau libre du fromage) entraîne un gonflement des caséines et une augmentation de la contrainte de rupture (Walstra et Van Vliet, 1982). L'affinage modifie la consistance du fromage et les produits de dégradation des caséines solubles dans l'eau ne participent plus à la construction de la matrice protéique (Walstra et Van Vliet, 1982). Par conséquent, plus la proportion d'azote soluble augmente plus la 
contrainte à la rupture diminue. Les fromages $\beta$-Cn $C$ ont, en général, un rapport humidité/caséine plus faible que les fromages $\beta$-Cn A (ce que l'on retrouve dans les images de structure) et une protéolyse plus limitée, ils sont aussi plus fermes. Les résultats confirment donc ceux de la bibliographie et montrent, en outre, une assez bonne cohérence entre la structure et la rhéologie des fromages. II faut cependant rappeler que la structure des minifromages n'a été analysée qu'à partir de 2 échantillons et que la relation structure-texture doit être traitée avec prudence car à côté de la matrice protéique du grain de caillé, la "cohésion" et la structure entre grains est également importante à considérer (Langley et Green, 1989).

II existe également une assez bonne concordance entre les résultats des mesures rhéologiques et ceux de l'analyse sensorielle, comme en témoignent les coefficients de corrélation entre les paramètres rhéologiques et les descripteurs de texture.

La description sensorielle des minifromages correspond bien à celle de fromages type beaufort. Les fromages ont une pâte souple à sableuse, sans ouverture. Ils ne présentent pas d'amertume (défaut rare en Beaufort), ont une saveur douce et un goût fruité (Chamba et al, 1994). Les principaux descripteurs de goût, les caractères piquant et fruité, sont corrélés à des indicateurs de la protéolyse. La protéolyse intervient en libérant des composés d'arôme qui étaient précédemment liés aux protéines (Mc Gugan et al, 1979). Berdagué et Grappin (1988) ont montré que la fraction NSAPT est corrélée au caractère fruité du gruyère de Comté. Aucune relation entre la fraction NSAPT et les descripteurs sensoriels n'apparaît ici mais les fromages ont tous le même âge. Le goût piquant, caractéristique de l'emmental français "grand-cru", est lié à des indicateurs d'une protéolyse avancée (Berdagué et al, 1990), ce qui ne semble pas le cas dans nos minifromages, où il apparaît plutôt lié aux particularités du variant $C$ de la caséine $\beta$. Biede et Hammond (1979) ont montré que la saveur douce est typique des gruyeres et il semble, comme le soulignent ces auteurs, qu'elle soit corrélée à la richesse en calcium des fromages.

\section{CONCLUSION}

Les minifromages de Beaufort fabriqués à partir de laits individuels de vaches Tarentaises d'haplotypes $\alpha_{s 1}-C n B$; $\beta-C n A$; $\kappa-C n$ A ou $\alpha_{s 1}-C n B ; \beta-C n C$; $\kappa-C n A$ sont différents. Les différences résultent des caractéristiques des laits mis en œuvre, de leur aptitude à la transformation fromagère et des mécanismes d'affinage des fromages à pâte cuite pressée. Lors de l'affinage, les produits d'hydrolyse des 2 variants de la $\beta$ $\mathrm{Cn}$, par la plasmine, sont différents. Les différences initiales de structure des caillés et de teneur en matière grasse des fromages (Delacroix-Buchet et Marie, 1994) confèrent aux fromages des structures différentes. Après 8 semaines d'affinage, les fromages n'ont pas les mêmes caractères rhèologiques et gustatifs. Les fromages $\beta-C n C$ comparés aux fromages $\beta-C n$ A sont moins élastiques, plus fermes et présentent plus souvent un goût piquant.

Cette étude montre qu'une race de vache, par ses caractéristiques génétiques, peut participer aux caractéristiques d'un fromage d'Appellation d'origine et que le variant $C$ de la caséine $\beta$ pourrait être considéré comme un marqueur de l'authenticité du fromage de Beaufort. Cependant, il semble qu'au point de vue de la transformation, le variant $C$ de la caséine $\beta$ n'apporte pas d'éléments favorables dans l'aptitude fromagère des laits de vaches Tarentaises et qu'il serait par conséquent peu judicieux de chercher à augmenter sa fréquence par la sélection. 


\section{REMERCIEMENTS}

Ce travail a reçu une aide financière du GIS Alpes-du-Nord. Nous remercions A Hauwuy (SUACI Alpes-du-Nord, 73000 Chambéry) qui a organisé et supervisé la collecte des laits de Savole, ainsi que nos collègues de l'INRA de Jouy-en-Josas, G Pitel pour les fabrications fromagères, M Rousseau, pour les observations au microscope électronique et l'interprétation des clichés photographiques et $D$ Le Bars pour sa précieuse collaboration lors des analyses chromatographiques. Nous tenons également à remercier JC Collin et O Rolet-Repecaud (INRA de Poligny) de nous avoir accueillis dans leur laboratoire pour réaliser les dosages immunochimiques de plasmine ainsi que S Fournier (ITG Centre, 39800 Poligny) de nous avoir fourni le profil électrophorétique permettant de comparer le fromage de Beaufort à l'emmental et au comté. Enfin, nous remercions tout particulièrement CM Papoff (universitè de Sassari, Sardaigne), en stage à l'INRA de Jouy-en-Josas, pour sa précieuse contribution à l'étude de l'hydrolyse par la plasmine des variants de la caséine $\beta$ et L Vassal (INRA de Jouy-en-Josas) pour sa relecture critique du manuscrit.

\section{RÉFÉRENCES}

AFNOR (1972) Guide pour l'implantation d'un local destiné aux analyses sensorielles. Norme NF V09-105

Barry JG. Donnelly WJ (1980) Casein compositional studies. I. The composition of casein from Friesian herd milks. J Dairy Res 47, 71-81

Benslimane S, Dognin-Bergeret MJ, Berdagué JL, Gaudemer $Y(1990)$ Variation with season and lactation of plasmin and plasminogen concentrations in Montbeliard cow's milk. J Dairy Res 57, 423-435

Berdagué JL, Grappin R (1988) Affinage et qualité du Gruyère de Comtè. IV. Caractéristiques sensorielles des fromages. Lait 68, 189-204

Berdagué JL, Grappin R, Chaillet B, Clément JF (1990) Caractérisation de l'emmental français agrand crum, II. Analyses sensorielles. Lait 70, 133-145

Biede SL, Hammond EG (1979) Swiss cheese flavor. 11. Organóleptic analysis. J Dairy Sci 62, 238-248

Chamba JF, Delacroix-Buchet A, Berdagué JL, Clément JF (1994) Une approche globale de la caractérisation des fromages : l'exemple du fromage de Beaufort. Sci Aliments 14, 585-594
Chen AH, Larkin JW, Clark CJ, Irwin WE (1979) Texture analysis method. J Dairy Sci 62, 901-907

Collin JC, Berdagué JL, Dognin-Bergeret M, Grappin A (1987) Affinage et qualité du Gruyère de Comté. IV. Étude de la protéolyse. Lait 67, 299-318

Collin JC, Le Bars D, Ryba I, Baer A (1990) Comparative measurements of the plasminogen-plasmin system of milk. XXIIIth Int Dairy Congr, Brief communications and Abstracts of posters, Montreal, 417

Corradini C, Battistotti B (1973) Indagine elettroforetica della progressiva idrolise delle proteine durante la maturazione del formaggio montasio. Sci Tec LattCasearia 24, 11-22

Delacroix-Buchet A, Marie C (1994) Comparaison des variants $A$ et $C$ de la caséine $B$ des laits de vaches Tarentaises en fabrication de fromage de type beaufort. I. Aptitudes fromagères et rendements en frais. Lait 74, 343-360

Delacroix-Buchet A, Trossat P (1991) Protéolyse et texture des fromages à pâte cuite pressée. I. Iniluence de l'activité de l'eau. Lait 71, 299-311

Desmazeaud MJ, Gripon JC, Le Bars D, Bergère JL (1976) Etude du rôle des microorganismes et des enzymes au cours de la maturation des fromages. III. Influence des microorganismes, Lait 56, 379-396

Gordon WG, Groves ML, Greenberg R, Jones SB, Kalan EB, Peterson RF, Townend RE (1972) Probable identification of $\gamma$-, TS-, R-, and S-caseins as fragments of $\beta$-casein. J Dairy Sci 55, 261-263

Gripon JC, Desmazeaud MJ, Le Bars D, Bergère JL (1975) Étude du rôle des microorganismes et des enzymes for a cours de la maturation des fromages. II. Influence de la présure commerciale. Lait 55, 502513

Grosclaude F (1988) Le polymorphisme génétique des principales lactoprotéines bovines. INRA Prod Anim 1, 5-17

Groves M, Gordon WG, Kalan EB, Jones SB (1972) Composition of bovine $\gamma$-caseins $A^{1}$ and $A^{3}$ and further evidence for a relationship in biosynthesis of $\gamma$ and $\beta$-caseins. J Dairy Sci 55, 1041-1049

Grufferty MB, Fox PF (1988) Milk alkaline proteinase. J Dairy Res 55, 609-630

Kaminogawa S, Yamauchi K (1972) Decomposition of $\beta$ casein by milk protease. Similarity of the decomposed products to temperature-sensitive and R-caseins. Agric Biol Chem 36, 255-260

Kerjean JR, Buisson V, Courroye M (1987) Miniaturisation de la fabrication fromagère. Tech Lait Market $1024,17-23$

Knoop AM, Buchheim W (1980) Die unterschiedliche Entwicklung der Substruktur bei der Reifung von Harzer-, Tilsiter- und Camembert-Käse. Milchwissenschaft $35,482-488$

Langley KR, Green ML (1989) Compression strength and fracture properties of model particulate food 
composites in relation to their microstructure and particle-matrix interaction. J Texture Stud 20, 191207

Lawrence RC, Creamer LK, Olson NF (1982) Rheological evaluation of maturing Cheddar cheese. J Food Sci 47, 631-646

Lawrence RC, Gilles J, Creamer LK (1983) The relationship between cheese texture and flavour. $N Z J$ Dairy Sci Technol 18, 175-190

Lawrence RC, Creamer LK, Gilles J (1987) Texture development during cheese ripening. J Dairy Sci 70, 1748-1760

Le Bars D, Gripon JC (1989) Specificity of plasmin towards bovine $\alpha_{\mathrm{s} 2}$-casein. J Dairy Res 56,817 821

Mc Gugan WA, Emmons DB, Larmond E (1979) Influence of volatile and non-volatile fractions on intensity of Cheddar cheese flavour. J Dairy Sci 62, 398-403
Nuyts-Petit V (1991) Influence des variants génétiques des caséines bovines sur l'aptitude fromagère du lait de vaches de races traditionnelles. Thèse de doctorat, université de Compiègne

Papoff CM, Delacroix-Buchet A, Le Bars D, Campus RI, Vodret $A$ (1994) Hydrolysis of bovine $C \beta$-casein by plasmin. Int J Food Sci (accepté pour publication)

Rüegg M, Eberhard P, Moor U, Flückiger E, Blanc B (1980) Beziehungen zwischen Teigheshaffenheit und Zuzammensetzung von Käse. Schweiz Milchw Forshung 9, 3-8

Schaar J (1985) Plasmin activity and proteose-peptone content of individual milks. J Dairy Res 52, 369-378

Steffen C (1975) Teigeigenschaften und Teigfehler um Emmentaler Käse. Schweiz Milchztg 104, 72-76

Vassal L (1984) L'analyse sensorielle du fromage. In: Le fromage (Eck A, ed). Lavoisier, Paris

Walstra P, van Vliet T (1982) Rheology of cheese. Bull Fed Int Lait 153, 22-27 